BRAVILLIAN JOURNAL

www.bjournal.com.br
ISSN 0100-879X

Volume 43 (3) 182-267 March 2011

BIOMEDICAL SCIENCES

AND

CLINICAL INVESTIGATION

Braz J Med Biol Res, March 2011, Volume 44(3) 263-266

doi: 10.1590/S0100-879X2011007500009

Effect of maternal hydration on the increase of amniotic fluid index

V.T.M. Borges, J. Rososchansky, J.F. Abbade, A. Dias, J.C. Peraçoli and M.V.C. Rudge

The Brazilian Journal of Medical and Biological Research is partially financed by

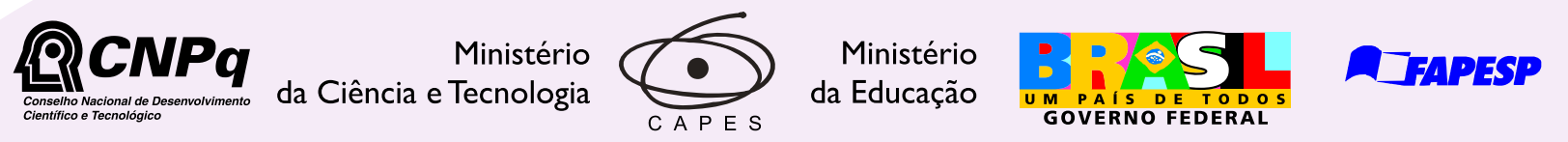

Institutional Sponsors
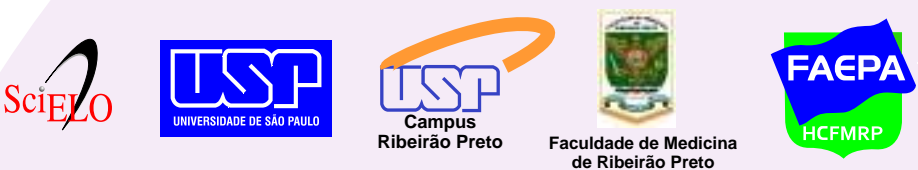

Ministério 


\title{
Effect of maternal hydration on the increase of amniotic fluid index
}

\author{
V.T.M. Borges ${ }^{1}$, J. Rososchansky¹, J.F. Abbade ${ }^{1}$, A. Dias ${ }^{2}$, J.C. Peraçoli ${ }^{1}$ \\ and M.V.C. Rudge 1
}

1Departamento de Obstetrícia e Ginecologia, 2Departamento de Saúde Pública, Faculdade de Medicina de Botucatu, Universidade Estadual Paulista "Julio Mesquita Filho",

Botucatu, SP, Brasil

\begin{abstract}
The objective of this study was to determine the effect of maternal hydration with oral isotonic solution and water on the amniotic fluid (AF) index of women with normohydramnios. Women with a normal AF index and gestational age between 33 and 36 weeks without maternal complications were randomized into three groups [isotonic solution (Gatorade ${ }^{\circledR}$ ), water, control]. The isotonic solution and water groups were instructed to drink $1.5 \mathrm{~L}$ of the respective solution and the control group was instructed to drink $200 \mathrm{~mL}$ water over a period of 2 to $4 \mathrm{~h}$. AF index was measured before and after hydration by Doppler ultrasonography. The investigator performing the AF index measurement was blind to the subject's group. Ninety-nine women completed the study without any adverse maternal effects. The median increase in AF index after hydration was significantly greater for the isotonic solution and water groups than for the control group. There was no significant difference between the isotonic solution and water groups. Hydration with isotonic solution and water caused a 10 -fold (95\% Cl: $2.09-49.89)$ and 6-fold $(95 \% \mathrm{Cl}: 1.16-30.95)$ increase in the chance of a $20 \%$ increase of AF index, respectively. Maternal hydration with isotonic solution or water increased the AF index in women with normohydramnios.
\end{abstract}

Key words: Amniotic fluid index; Maternal oral hydration; Isotonic solution

\section{Introduction}

Adequate amniotic fluid (AF) volume is considered to be important for fetal well-being $(1,2)$. The determination of an association between oligohydamnios and poor fetal outcome requires the investigation of the factors involved in the maintenance of AF volume, and maternal hydration, among these, seems to play a relevant role (3).

Several studies have addressed the relationship between maternal intravascular volume and AF volume. Maternal hydration has been the focus of studies in women with oligohydramnios. In two studies, Kilpatrick et al. (2) and Kilpatrick and Safford (4) reported that maternal water hydration increased the AF index both in pregnancies with oligohydramnios and in those with a normal AF index (4).

An alternative route of maternal hydration is intravenous fluid loading. Dói et al. (5) compared the effects of maternal hydration with oral water, intravenous hypotonic fluid and isotonic fluid on AF volume in women with oligohydramnios and found an increase of AF index only in the hypotonic fluid and water groups.
A systematic review of the literature demonstrated that simple maternal hydration appears to increase AF volume and may be beneficial in the prevention or management of oligohydramnios during labor or prior to external cephalic version. However, the authors concluded that controlled trials are needed to assess the clinical benefits and possible risks of maternal hydration for specific clinical purposes (6).

Drinking too much water may not be pleasant for some people due to the characteristics of water, and therefore the isotonic solution (Gatorade ${ }^{\circledR}$ ), which can have different flavors, seems to be a more pleasing alternative to oral hydration.

No reported studies on women with normal AF have compared the effects of oral hydration with isotonic fluid and water on AF index. The present study, therefore, was designed to compare the effects of maternal oral hydration with isotonic fluid and water on AF index in women with normohydramnios.

Correspondence: V.T.M. Borges, Departamento de Obstetrícia e Ginecologia, Faculdade de Medicina de Botucatu, UNESP, Distrito de Rubião Junior, s/n, Caixa Postal 530, 18618-970 Botucatu, SP, Brasil. E-mail: vborges@fmb.unesp.br

Received May 22, 2010. Accepted January 3, 2011. Available online January 21, 2011. Published March 7, 2011. 


\section{Subjects and Methods}

This blind, randomized trial was approved by the Institutional Review Board of Botucatu Medical School, São Paulo State University, UNESP, Brazil, and each subject gave written informed consent to participate.

The inclusion criteria were gestational age between 33 and 36 weeks, singleton pregnancy, intact membranes, initial AF index between 5 and 95\% according to Moore and Cayle (7), no maternal complications, no structural fetal malformation, and no evidence of fetal distress in a nonstress test.

Based on a study by Kilpatrick et al. (2) using the same methodology, a sample size calculation, with $\alpha=0.05$ and $\beta=20 \%$, and effect size of a $20 \%$ increase in AF index, indicated that approximately 29 subjects were needed per group.

An investigator who had not determined the AF index randomized each subject to the isotonic group (Gatorade ${ }^{\circledR}$; $\mathrm{N}=34)$, water group $(\mathrm{N}=30)$ or control group $(\mathrm{N}=35)$ using opaque envelope selection. The envelopes, each one containing the group notation, had been randomly mixed and then numbered consecutively. The isotonic and water groups of pregnant women ingested $1.5 \mathrm{~L}$ of the respective solution and the control group ingested $200 \mathrm{~mL}$ water. The isotonic solution and the water were presented to the subjects at $4^{\circ} \mathrm{C}$.

After an initial determination of the AF index, each woman was instructed to drink the fluid over a period of 2 to $4 \mathrm{~h}$ and to return for a second determination of the AF index 2-5 $\mathrm{h}$ after finishing the solution. Subjects were asked not to reveal to which group they had been randomized. The times of the pre-treatment and post-treatment AF indexes were recorded for all women, and the women in the isotonic solution and water groups recorded the time they began drinking and the time they finished drinking the solutions. All subjects were asked to write down the volume of liquid they had consumed the previous day for an estimate of background hydration.

$\mathrm{AF}$ indexes were measured with a 3.5-MHz transducer with pulse-wave color Doppler ultrasonography by an ultrasonographer who was blind to the randomization of the study groups. The AF index was measured by the method of Phelan et al. (8). Two AF indexes were measured during each visit and the means were used for analysis.

Within-group data were compared by the unpaired Wilcoxon test. The differences between prehydration and posthydration values are presented as the delta and were compared between groups by the unpaired Kruskal-Wallis test. The level of significance was set at the level of $P<$ 0.05 in all analyses. The statistical SAS package, version 8.2-2001, was used to analyze the data. The odds ratio was calculated using the $95 \%$ confidence interval, considering a $20 \%$ improvement of the AF index, in order to compare the outcomes of the three groups using the Epi-Info- 6 statistical package.

\section{Results}

The demographic characteristics of the study groups are listed in Table 1. Maternal age, gestational age and parity were similar for all groups.

There was no difference between groups in mean fluid intake on the previous day, with a mean of approximately $1.9 \mathrm{~L}$ each. There were no differences in the time between examinations, with $366 \pm 71,349 \pm 48$, and $341 \pm 45 \mathrm{~min}$ for the isotonic, water and control groups, respectively. There was no difference between the isotonic and water groups in the mean time to drink the solution. There was no change in the mean time between finishing the solution and the determination of the post-treatment AF index for the isotonic and water groups.

The median pre- and post-treatment AF indexes did not differ significantly between groups, although oral hydra-

Table 1. Demographic characteristics of the three groups studied.

\begin{tabular}{lccc}
\hline Characteristic & Isotonic solution $(\mathrm{N}=34)$ & Water $(\mathrm{N}=30)$ & Control $(\mathrm{N}=35)$ \\
\hline Maternal age (years) & $24(8.5)$ & $26(12.5)$ & $20(10)$ \\
Gestational age (weeks) & $35(1.5)$ & $35.4(1.6)$ & $34.4(2.2)$ \\
Parity (\%) & & & \\
1 & 76.5 & 66.7 & 65.7 \\
2 & 2.9 & 10.0 & 22.9 \\
3 & 14.7 & 16.7 & 5.7 \\
4 & 2.9 & 3.3 & 5.7 \\
5 & 2.9 & - & - \\
7 & - & 3.3 & - \\
\hline
\end{tabular}

Data are reported as medians and interquartile ranges and percentage. There were no statistically significant differences between groups (unpaired Kruskal-Wallis test). 
tion with isotonic solution or water increased the AF index significantly (Table 2). The median AF index of the control group decreased from pre- to post-treatment (Figure 1).

Table 3 shows that hydration with isotonic solution and water increased by 10- and 6-fold, respectively, the chance of a $20 \%$ improvement of the AF index compared to control.

\section{Discussion}

The present randomized study demonstrated that in women with normal AF index, a significant increase in AF index was achieved by oral hydration with both isotonic solution and water. This is the first study, which compared oral hydration with isotonic solution and water in women with a normal AF index. These results agree with the existing evidence for the role of maternal hydration in AF dynamics.

Our results were similar to those obtained in previous studies (2,4-6). Kilpatrick et al. (2) demonstrated a significant increase in AF index (approximately $30 \%$ ) in women with oligohydramnios, 2-4 $\mathrm{h}$ after water hydration. Repeating the study in women with a normal AF index, they reported an increase of $3 \mathrm{~cm}(16 \%)$ in AF index with water (4). Dói et
Table 2. Pre- and post-treatment amniotic fluid index (AFI) in the three groups studied.

\begin{tabular}{ccccc}
\hline & $\begin{array}{c}\text { Isotonic solution } \\
(\mathrm{N}=34)\end{array}$ & $\begin{array}{c}\text { Water } \\
(\mathrm{N}=30)\end{array}$ & $\begin{array}{c}\text { Control } \\
(\mathrm{N}=35)\end{array}$ & $\mathrm{P}^{*}$ \\
\hline $\mathrm{AFI}(\mathrm{mm})$ & & & & \\
Pre-treatment & $142.25(51.93)$ & $162.05(54.18)$ & $152.20(58.00)$ & $\mathrm{NS}$ \\
Post-treatment & $156.30(66.25)$ & $162.15(76.90)$ & $149.50(53.50)$ & $\mathrm{NS}$ \\
$\mathrm{P}^{* *}$ & $<0.001$ & $<0.001$ & $<0.001$ & \\
\hline
\end{tabular}

Data are reported as medians and interquartile ranges. NS = non-significant. *Kruskal-Wallis test. ${ }^{*}$ Wilcoxon test.
Table 3. Odds ratio for a $20 \%$ increase in amniotic fluid index in the three groups studied.

\begin{tabular}{lcl}
\hline & OR & \multicolumn{1}{c}{$95 \% \mathrm{Cl}$} \\
\hline Isotonic solution vs control & 10.21 & $2.09-49.89$ \\
Water vs control & 6.01 & $1.16-30.95$ \\
Isotonic solution vs water & 1.71 & $0.58-4.94$ \\
\hline
\end{tabular}

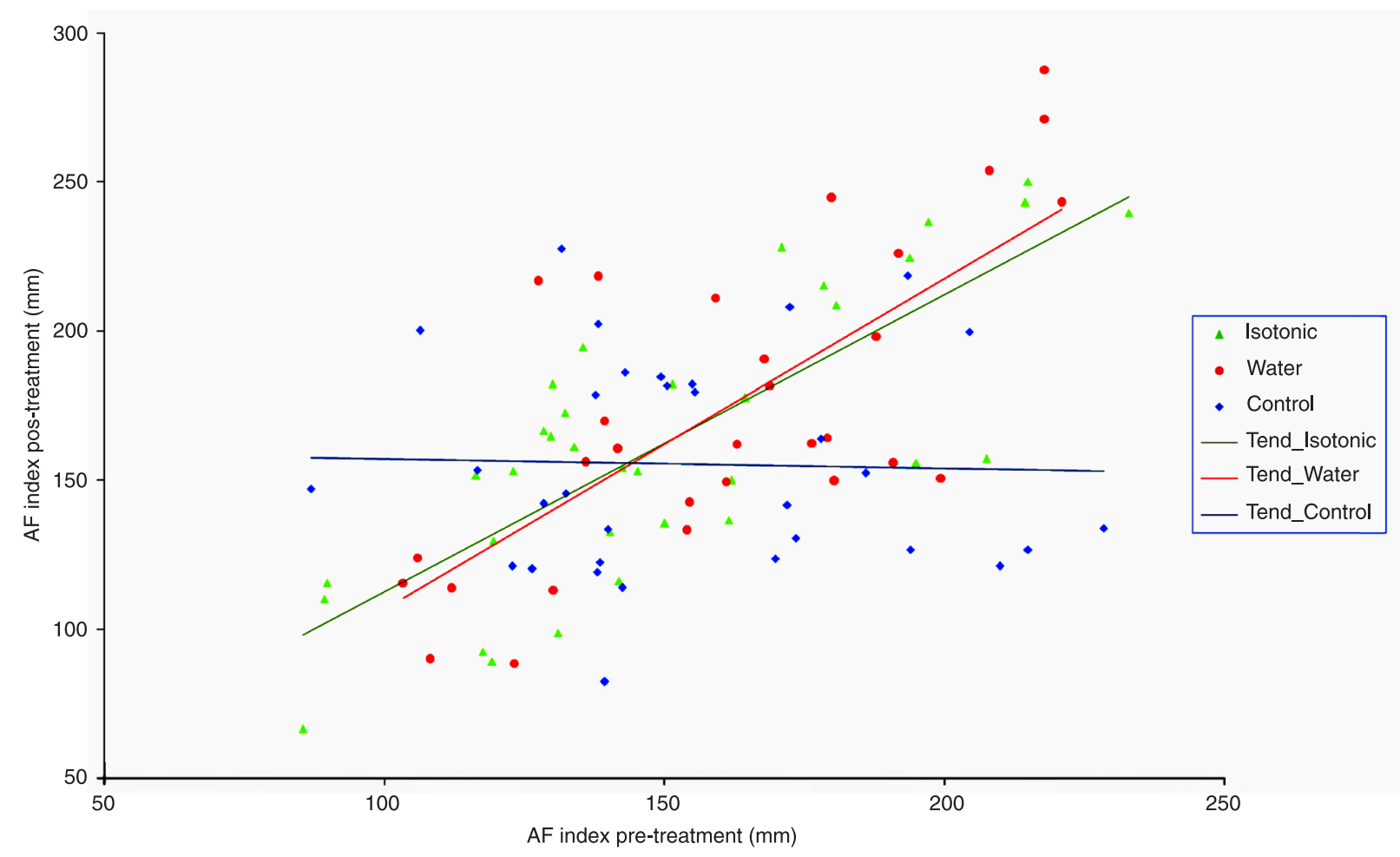

Figure 1. Scatterplot of pre- and post-treatment amniotic fluid index according to group. 
al. (5) demonstrated an increase in AF index in women with oligohydramnios after hydration with intravenous infusion of hypotonic fluid or with oral water.

The present study was carried out by the regular staff of a public tertiary level university hospital in Brazil. It is important to mention that Brazil is a tropical country of warm climate and that maternal hydration with isotonic solution is another option of low-cost and acceptable treatment justified by the fact that the benefits are greater than the costs. In Brazil, the cost of isotonic solution is about US\$3.50/1.5 L.

Although maternal hydration increased the AF index, neither the mechanism responsible for this change nor the length of time the increase would persist is clear. This trial was not designed to answer these questions, but to determine whether the AF index could be increased with oral hydration. Although it is not known why AF is reduced in some pregnancy states, it is accepted that fetal urine output is a major contributor to AF volume after the 24th week of pregnancy. In adults, diuresis is directly correlated with intravascular volume and osmolality. There are clinical data suggesting that the fetus can respond to a maternal change in either intravascular volume or osmolality (9-11).

Goodlin et al. (12) reported a correlation between measured maternal intravascular volume and AF volume.

\section{References}

1. Mauad Filho F, Ayres CE, Ferreira AC, Paton MRF, Baracchini JAA, Casillo PM. The amniotic fluid volume in pregnant women under subtotal immersion in water. Rev Bras Ginecol Obstet 1996; 18: 297-302.

2. Kilpatrick SJ, Safford KL, Pomeroy T, Hoedt L, Scheerer L, Laros RK. Maternal hydration increases amniotic fluid index. Obstet Gynecol 1991; 78: 1098-1102.

3. Moore TR. Clinical assessment of amniotic fluid. Clin Obstet Gynecol 1997; 40: 303-313.

4. Kilpatrick SJ, Safford KL. Maternal hydration increases amniotic fluid index in women with normal amniotic fluid. Obstet Gynecol 1993; 81: 49-52.

5. Dói S, Osada H, Seki K, Sekiya S. Effect of maternal hydration on oligohydramnios: a comparison of three volume expansion methods. Obstet Gynecol 1998; 92: 525-529.

6. Hofmeyr GJ, Gülmezoglu AM. Maternal hydration for increasing amniotic fluid volume in oligohydramnios and normal amniotic fluid volume (Cochrane Review). The Cochrane library. Issue 2. [Computer program]. Oxford: Update Software; 2005.

7. Moore TR, Cayle JE. The amniotic fluid index in normal human
Experimental data show that the fetal urine output changes with alterations in maternal osmolality. Water deprivation or mannitol infusion resulted in a decrease in fetal urine flow in ewes, implicating maternal osmolality as an important variable $(9-11,13)$. However, it is also clear that changes in fetal volume or osmolality can alter fetal urine output, AF volume and fetal intravascular volume (10). In the present study, although maternal osmolality and intravascular volume were not measured, we believe that the change in AF index induced by the isotonic solution may be justified by maternal intravascular volume expansion. Regarding water hydration, since it is a hypotonic solution, this alteration might be due to osmolality.

Maternal oral hydration with isotonic solution or with water increases the AF index in women with a normal AF volume and the lack of maternal hydration for several hours reduced the AF index in tropical countries. Our results can be used in systematic reviews because they were obtained in a randomized trial, with no violation of the position of the groups or exclusion after randomization. However, more controlled and randomized trials are needed to assess the clinical benefits and possible risks of maternal hydration for special clinical purposes in other countries with lower environmental temperatures. pregnancy. Am J Obstet Gynecol 1990; 162: 1168-1173.

8. Phelan JP, Ahn MO, Smith CV, Rutherford SE, Anderson E. Amniotic fluid index measurements during pregnancy. $J$ Reprod Med 1987; 32: 601-604.

9. Stevens $A D$, Lumbers ER. The effect of maternal fluid intake on the volume and composition of fetal urine. J Dev Physiol 1985; 7: 161-166.

10. Brace RA. Amniotic fluid volume and its relationship to fetal fluid balance: review of experimental data. Semin Perinatol 1986; 10: 103-112.

11. Lumbers ER, Stevens AD. Changes in fetal renal function in response to infusions of a hyperosmotic solution of mannitol to the ewe. J Physiol 1983; 343: 439-446.

12. Goodlin RC, Anderson JC, Gallagher TF. Relationship between amniotic fluid volume and maternal plasma volume expansion. Am J Obstet Gynecol 1983; 146: 505-511.

13. Battaglia F, Prystowsky H, Smisson C, Hellegers A, Bruns P. Fetal blood studies. XIII. The effect of the administration of fluids intravenously to mothers upon the concentrations of water and electrolytes in plasma of human fetuses. Pediatrics 1960; 25: 2-10. 